\title{
Efficacy and Safety of SGLT2 Inhibitors in Reducing Glycated Hemoglobin and Weight in Emirati Patients With Type 2 Diabetes
}

\author{
Alaaeldin Bashier ${ }^{\mathrm{a}, \mathrm{e}}$, Azza Abdulaziz Khalifa ${ }^{\mathrm{a}}$, Fauzia Rashid ${ }^{\mathrm{a}}$, Elamin Ibrahim Abdelgadir ${ }^{\mathrm{a}}$, \\ Amina Adil Al Qaysi ${ }^{b}$, Razan Ali ${ }^{b}$, Ahmed Eltinayc, Jalal Nafach ${ }^{\mathrm{d}}$, \\ Fatima Alsayyaha ${ }^{\mathrm{a}}$, Fatheya Alawadi ${ }^{\mathrm{a}}$
}

\begin{abstract}
Background: SGLT2 inhibitors are a new class of drugs that act by inhibiting glucose reabsorption in the proximal renal tubules. Many trials have demonstrated their effectiveness in reducing glycated hemoglobin (HbAlc) and weight, but they have never been examined in Arab or Emirati populations.

Methods: We assessed the efficacy of SGLT2 inhibitors in reducing $\mathrm{HbAlc}$ and weight in our population and specifically in an Emirati cohort. We also assessed the effect on fasting blood glucose, blood pressure, lipid profile, serum creatinine, and side effects.

Results: The total number of patients was 307 . The baseline HbA1c in the Emirati cohort was $8.9 \pm 1.7 \%$, which dropped significantly to $8 \pm 1.5 \%$ at 6 months $(\mathrm{P}=0.0001)$. At 1 year, the mean $\mathrm{HbAlc}$ was $8 \pm 1.4 \%$, which was significantly different from baseline $(\mathrm{P}=0.0001)$. However, the change in mean HbAlc from 6 months $(8 \pm 1.5 \%)$ to 1 year $(8 \pm 1.4 \%)$ was not statistically significant $(\mathrm{P}=0.88)$. A similar highly significant change was observed when comparing weights at baseline and 6 months in the Emirati population $(85.7 \pm 17.8 \mathrm{~kg}$ vs. $84 \pm 17.2 \mathrm{~kg}, \mathrm{P}=0.0001)$. Total cholesterol dropped significantly at 6 months $(\mathrm{P}=0.008)$, as did low-density lipoprotein $(\mathrm{LDL})(\mathrm{P}=0.005)$.

Conclusions: The use of SGLT2 inhibitors is associated with significant reductions in $\mathrm{HbAlc}$ and weight. Unlike all previous trials, the inhibitors significantly reduced total cholesterol and LDL. Larger trials are needed to reassess their effects on lipid parameters.
\end{abstract}

Keywords: SGLT2 inhibitors; Emirati; Efficacy; Weight reduction; Canagliflozin; Dapagliflozin; HbA1c

\footnotetext{
Manuscript accepted for publication March 09, 2017

${ }^{a}$ Endocrine Department, Dubai Hospital, Dubai, United Arab Emirates b'Internal Medicine Residency Program, Dubai Hospital, Dubai, United Arab Emirates

'Emergency Medicine Residency Program, Rashid Hospital, Dubai, United Arab Emirates

${ }^{d}$ Dubai Diabetes Centre, Dubai, United Arab Emirates

${ }^{e}$ Corresponding Author: Alaaeldin M. K. Bashier, Endocrine Department, Dubai Hospital, Dubai, United Arab Emirates.Email: alaaeldin11@gmail.com
}

doi: https://doi.org/10.14740/jocmr2976w

\section{Introduction}

SGLT2 inhibitors are a new class of drugs that act with an insulin-independent mechanism. They inhibit reabsorption of filtered glucose by blocking the SGLT2 transporters in the proximal renal tubule, which results in excess glucose loss in urine. All initial trials with SGLT2 inhibitors were shown to be very effective in reducing glycated hemoglobin ( $\mathrm{HbAlc}$ ), with an average reduction of $\mathrm{HbA} 1 \mathrm{c}$ by $0.6-1.2$ depending on the baseline level [1]. SGLT2 inhibitors also result in an average weight loss of approximately $2 \mathrm{~kg}$ compared with placebo, irrespective of the presence or type of concomitant anti-diabetes therapy [2]. Weight loss becomes evident after 6 weeks and then usually reaches a plateau or slightly rebounds after 26 - 32 weeks [3]. Other beneficial effects include a reduction in blood pressure (BP), with many studies showing average reductions in systolic blood pressure (SBP) of $4 \mathrm{~mm} \mathrm{Hg}$ and in average diastolic blood pressure (DBP) of $1.6 \mathrm{~mm} \mathrm{Hg}$ [4]. Only canagliflozin showed a dose-response relationship with systolic blood pressure [4].

Two recent major trials have shown that SGLT2 inhibitors are superior to many other anti-diabetic medications in secondary prevention of cardiovascular events and renal protection. In the EMPA-REG trial, empagliflozin reduced death from cardiovascular causes (hazard ratio: 0.62 ; 95\% confidence interval (CI): $0.49-0.77 ; \mathrm{P}<0.001)$, death from any cause (hazard ratio: $0.68 ; 95 \%$ CI: $0.57-0.82$; P $<0.001$ ), and hospitalization for heart failure (hazard ratio: $0.65 ; 95 \%$ CI: $0.50-0.85 ; \mathrm{P}=0.002$ ) in patients with type 2 diabetes and a previous cardiovascular event [5]. In the EMPA-REG renal outcomes trial, empagliflozin reduced microalbuminuria, time to doubling of serum creatinine, time to initiation of renal replacement therapy, and death due to renal disease [6].

The efficacy and safety of SGLT2 inhibitors have been proved in Western populations and Asian populations. Yang et al conducted a pooled analysis from eight phase IIb/III double-blind trials with durations of up to 24 weeks examining treatments with placebo $(\mathrm{N}=497), 5 \mathrm{mg}$ of dapagliflozin ( $\mathrm{N}$ $=491)$, or $10 \mathrm{mg}$ of dapagliflozin $(\mathrm{N}=465)$ [7]. They demonstrated the safety, efficacy, and tolerability of dapagliflozin in an Asian population. Kaku et al assessed the safety and efficacy of dapagliflozin when used as a monotherapy after the failure of diet and exercise in Japanese patients. They showed greater reductions in mean HbA1c level with dapagliflozin (5 
$\mathrm{mg},-0.41 \% ; 10 \mathrm{mg},-0.45 \%)$ than with placebo $(-0.06 \%)$ at week 24. Furthermore, fasting plasma glucose (FPG) was also significantly reduced with dapagliflozin $(5 \mathrm{mg},-8.6 \mathrm{mg} / \mathrm{dL} ; 10$ $\mathrm{mg},-13.7 \mathrm{mg} / \mathrm{dL})$ compared with placebo $( \pm 5.8 \mathrm{mg} / \mathrm{dL})$. It significantly reduced body weight as well $(5 \mathrm{mg},-2.13 \mathrm{~kg} ; 10$ $\mathrm{mg},-2.22 \mathrm{~kg})$ compared with placebo $(-0.84 \mathrm{~kg})$ [8].

$\mathrm{Ji}$ et al also proved the efficacy as a monotherapy in a randomized double-blind controlled trial in which $89 \%$ of the patients were Chinese. At week 24, the mean reductions in $\mathrm{HbA} 1 \mathrm{c}$ were $-0.29 \%$ for the placebo group versus $-1.04 \%$ and $-1.11 \%$ for the 5- and 10-mg dapagliflozin groups, respectively $(\mathrm{P}<$ 0.0001 for both doses). Changes in FPG were 2.5, -25.1, and $-31.6 \mathrm{mg} / \mathrm{dL}(0.14,-1.39$, and $-1.75 \mathrm{mmol} / \mathrm{L})$ for placebo, $5 \mathrm{mg}$ of dapagliflozin, and $10 \mathrm{mg}$ of dapagliflozin, respectively [9].

In an interesting study, Gavin et al assessed canagliflozin's safety, efficacy, and effect on BP and lipid profile in different racial groups. The data were pooled from four placebo-controlled phase 3 studies and two placebo-controlled sub-studies of a population of patients with inadequately controlled type 2 diabetes (total number $=4,158$ ). Of these patients, $75 \%$ were white, $13 \%$ were Asian, $4 \%$ were black, and $8 \%$ were another race. Changes in $\mathrm{HbAlc}$, body weight, and SBP were similar in all racial groups. Genital mycotic infections were observed more often in black males and males of other races, whereas urinary tract infection and osmotic diuresis-related adverse events were less common in Asians [10].

There are no data on the efficacy and safety of SGLT2 inhibitors in Arab or Emirati populations. Therefore, we conducted this retro-prospective trial to assess their effects on $\mathrm{HbA1c}$, weight, BP, and lipid profiles. We also aimed to assess the safety of this class of drugs.

\section{Methodology}

\section{Study design}

We performed this multicenter, retrospective trial at two sites at the Dubai Health Authority (DHA) in Dubai. The trial protocol was reviewed and approved by the institutional review board and ethics committee of the DHA. We screened all patients with type 2 diabetes who received SGLT2 inhibitors in the period between April 2015 and February 2016 for eligibility for the study. The data were gathered and analyzed by the investigators. All the authors had access to the final results and vouch for the fidelity of the trial to the protocol. Some authors collaborated in writing the first draft of the manuscript, which was revised and approved by all the authors. All authors assume responsibility for the accuracy and completeness of the content and for the decision to submit the manuscript for publication. We sought editorial support that was provided by an on-line manuscript reviewer under guidance from the authors.

\section{Patients}

We screened all patients with type 2 diabetes who had been started on treatment with SGLT2 inhibitors in the period between April 2015 and February 2016 for eligibility for the trial. All prescriptions were compliant with the DHA protocols and recommendations, which means that none was pregnant, all were $>18$ years of age, and none of the patients had estimated glomerular filtration rate $(\mathrm{eGFR})<60 \mathrm{~mL} / \mathrm{min} / \mathrm{m}^{2}$. The primary reasons for exclusion were a mixed prescription of dapagliflozin and canagliflozin and incomplete data retrieval from the electronic database. The doses available in our institutions are $100 \mathrm{mg} /$ day for canagliflozin and $10 \mathrm{mg} /$ day for dapagliflozin.

\section{Procedures}

After screening patients for eligibility, we gathered data retrospectively for those who started treatment in 2015 . We excluded those who did not have complete data or had a prescription that included both canagliflozin and dapagliflozin at different times. We also excluded patients whose medications were adjusted during the initial 6-month period to minimize their effect on the studied parameters.

The electronic database was reviewed to look for baseline information. The information included patients' demographics (age, sex, and nationality), clinical characteristics (duration of diabetes, co-morbidities, medications used at baseline, BP, and weight), and laboratory results (fasting blood glucose (FBG), $\mathrm{HbAlc}$, lipid profile, and creatinine). All information was entered on a data collection sheet. We then collected the same data at 6 months. FBG and $\mathrm{HbA} 1 \mathrm{c}$ were also obtained at 1 year after starting the medicine.

Patients' medical charts were also reviewed to look for documented side effects (genital infections, urinary tract infections, severe hypoglycemia, admission with fracture, and acute renal failure) and to ensure that patients were compliant with treatment. This was also helpful for cross-checking and validating the data obtained from the electronic medical system. The data obtained were then reviewed by another investigator to ensure accuracy.

\section{Outcomes}

The primary objective was to assess the efficacy of SGLT2 inhibitors in reducing $\mathrm{HbA} 1 \mathrm{c}$ and weight in an Emirati population. As a secondary aim, we sought to determine the effects of the SGLT2 inhibitors on FBG, lipid profile, and BP at baseline and 6 months. We also assessed the safety of these medications by evaluating the change in serum creatinine from baseline and at 3 months of therapy, as well as the reported side effects.

\section{Definitions}

Patients were considered to have type 2 diabetes if they fulfilled the American Diabetes Association (ADA) criteria for the diagnosis of diabetes mellitus $(\mathrm{FBG} \geq 126 \mathrm{mg} / \mathrm{dL}$, random 


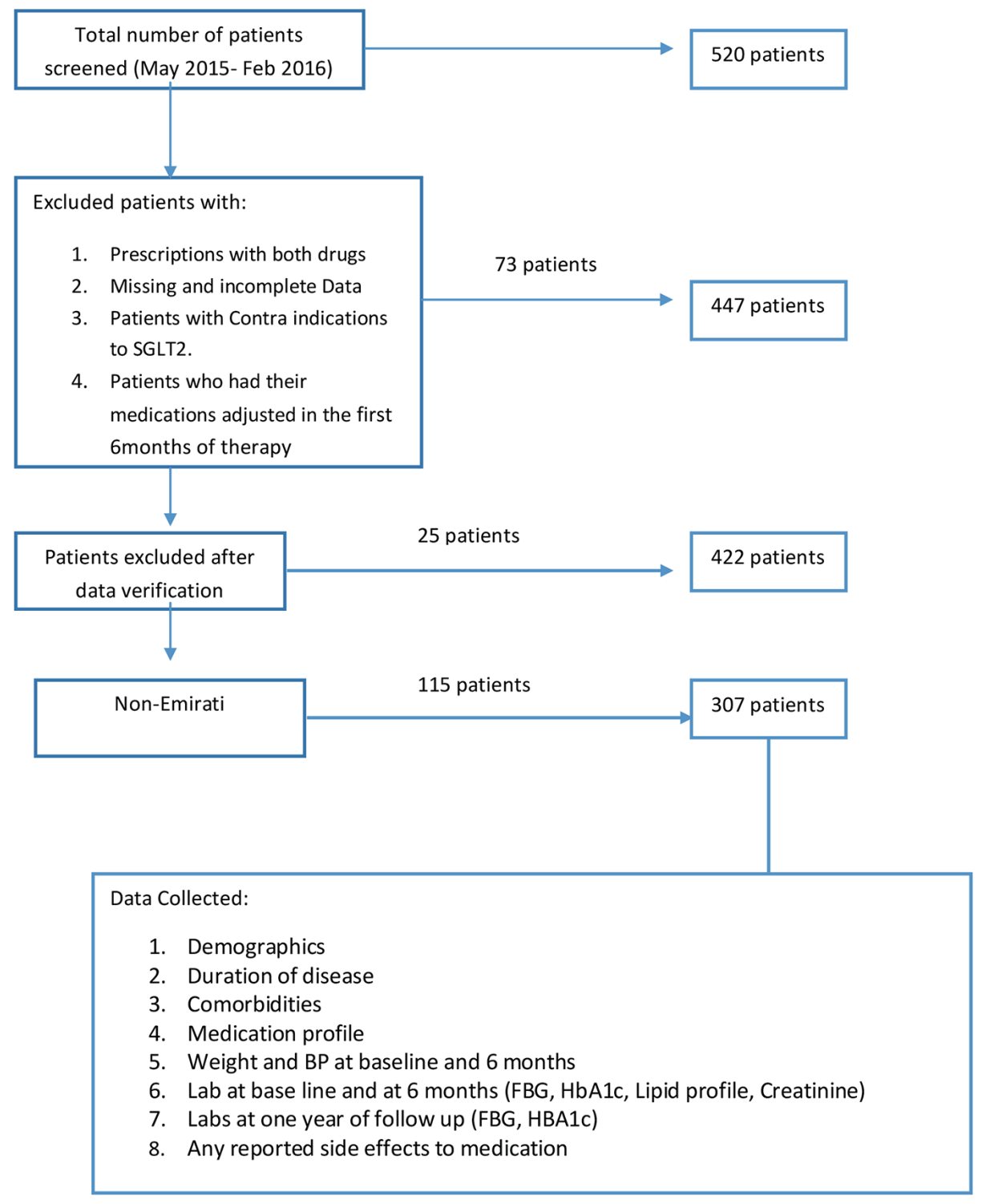

Figure 1. Study flow chart.

blood glucose $(\mathrm{RBG}) \geq 200 \mathrm{mg} / \mathrm{dL}$, or $\mathrm{HbA} 1 \mathrm{c} \geq 6.5 \%$ ) [10], or if they are already on anti-diabetic agents. However, we did not consider patients on metformin alone to be diabetic unless they fulfilled the ADA criteria, since many young women were on metformin for treatment of other medical problems, such as polycystic ovarian syndrome. We defined hypertension by an $\mathrm{SBP} \geq 140 \mathrm{~mm} \mathrm{Hg}$ and DBP $\geq 90 \mathrm{~mm} \mathrm{Hg}$ or the use of antihypertensive medications.

\section{Statistical analysis}

We based the required sample size for the trial on the latest census data on the population of Dubai and the prevalence of diabetes in the Emirati population published in the International Diabetes Foundation's most recent atlas. Assuming a
$90 \% \mathrm{CI}$ and $5 \%$ margin of error, we calculated that 267 patients would be needed to give the study the required statistical power. We performed the data analysis using SPSS software version 16.0. In all analyses, a P-value $<0.05$ was considered significant, and $\mathrm{P}<0.001$ was considered highly significant. Quantitative variables are described using the mean, SD, and range, while qualitative variables are defined using numbers and percentages.

We used the Chi-squared test to compare qualitative variables between groups. The unpaired $t$-test was used to compare quantitative variables in parametric data ( $\mathrm{SD}<50 \%$ mean), while the Mann-Whitney test was used for non-parametric data. We used a paired $t$-test to compare quantitative variables within the same group before and after the treatment for parametric data ( $\mathrm{SD}<50 \%$ mean), whereas the Wilcoxon test was used for parametric data $(\mathrm{SD}>50 \%$ mean). 


\section{Results}

\section{Overview of trial conduct}

A total of 520 patients who started treatment with one of the two available SGLT2 inhibitors in DHA (dapagliflozin $10 \mathrm{mg}$ and canagliflozin $100 \mathrm{mg}$ ) were initially screened. Of these, 73 patients were excluded for either changing between the two medications, incomplete data, or adjustment of the medications during the first 6-month period. Another 25 patients were further excluded after cross-checking data in files with the electronic database. An additional 115 patients who fulfilled all criteria were later excluded because they were not Emirati nationals. A total of 307 patients were included in the final analysis (Fig. 1).

Of the 307 patients, the majority were females $(n=194$, $63.2 \%$ ), and the mean age was $54.368 \pm 12.4$ years. At baseline, the mean duration of diabetes was 14.03 years, and the duration ranged between 3 months and 37 years. The mean $\mathrm{HbAlc}$ level was $8.9 \pm 1.7 \%$, and the mean weight was 85.5 $\pm 17 \mathrm{~kg}$ with a range of $48.5-143 \mathrm{~kg}$. The rest of the demographic and clinical characteristics are shown in Table 1.

\section{Glycemic control}

The baseline HbA1c was $8.9 \pm 1.7 \%$, which significantly dropped to $8 \pm 1.5 \%$ at 6 months $(\mathrm{P}=0.0001)$. At 1 year, the mean $\mathrm{HbAlc}$ was $8 \pm 1.4 \%$, which was significantly different from baseline $(\mathrm{P}=0.0001)$. However, the change in mean HbAlc from 6 months $(8 \pm 1.5 \%)$ to 1 year $(8 \pm 1.4 \%)$ was not statistically significant $(\mathrm{P}=0.88)$. Variations in the $\mathrm{HbAlc}$ values over time are shown in Figure 2. The difference between mean FBG in our Emirati patients at baseline and 6 months had high statistical significance $(P=0.0001 ; 174 \pm 61 \mathrm{mg} / \mathrm{dL}$ at baseline vs. $150 \pm 50 \mathrm{mg} / \mathrm{dL}$ at 6 months). However, there was no statistical difference when mean blood glucose at both baseline and 6 months were compared to that at 1 year (mean fasting glucose $159 \pm 66 \mathrm{mg} / \mathrm{dL} ; \mathrm{P}=0.1$ and 0.7 , respectively). The changes in mean $\mathrm{HbAl}$ c and FBG remained significant after correction for age, sex, duration of diabetes, comorbidities, and medication use at baseline.

\section{Weight changes}

The change in weight in our cohort was highly significant when baseline $(85.5 \pm 17 \mathrm{~kg})$ was compared to that at 6 months $(84.0 \pm 17.2 \mathrm{~kg}, \mathrm{P}=0.0001)$. Changes in weight are shown in Figure 3. Changes in mean weight remained significant after correction for age, sex, duration of diabetes, comorbidities, and medication use at baseline.

\section{Changes in BP and lipid profiles}

The change in SBP was significant in the Emirati patients (137 \pm
Table 1. Baseline Characteristics

\begin{tabular}{|c|c|c|}
\hline Variables & No & $\%$ \\
\hline \multicolumn{3}{|l|}{ Age } \\
\hline$\geq 65$ years & 67 & $21.8 \%$ \\
\hline$<65$ & 240 & $78.2 \%$ \\
\hline Mean \pm SD & $54.4 \pm 11.6$ & \\
\hline Weight & $85.3 \pm 16$ & $85-143$ \\
\hline \multicolumn{3}{|l|}{ Gender } \\
\hline Male & 113 & $36.8 \%$ \\
\hline Female & 194 & $63.2 \%$ \\
\hline \multicolumn{3}{|l|}{ Medications } \\
\hline Long acting & 114 & $37.1 \%$ \\
\hline Bolus & 82 & $26.7 \%$ \\
\hline Metformin & 280 & $91.2 \%$ \\
\hline DDP4 & 162 & $52.8 \%$ \\
\hline GLP & 124 & $40.4 \%$ \\
\hline SU & 143 & $46.6 \%$ \\
\hline TZD & 27 & $8.8 \%$ \\
\hline Statins & 280 & $91.2 \%$ \\
\hline Others & 35 & $11.4 \%$ \\
\hline \multicolumn{3}{|l|}{ Comorbidities } \\
\hline HTN & 232 & $75.6 \%$ \\
\hline Dyslipidemia & 222 & $72.3 \%$ \\
\hline Hypothyroidism & 24 & $7.8 \%$ \\
\hline IHD & 32 & $10.4 \%$ \\
\hline Others & 62 & $20.2 \%$ \\
\hline DM duration (years) & $14.03 \pm 6.6$ & 3 months -37 years \\
\hline SBP & $136 \pm 16$ & $90-219$ \\
\hline DBP & $74.2 \pm 19$ & $40-178$ \\
\hline FBG & $169 \pm 59$ & $45-395$ \\
\hline $\mathrm{HbA1C}$ & $8.7 \pm 1.6$ & $5-17$ \\
\hline $\mathrm{TC}$ & $164.8 \pm 42$ & $76-357$ \\
\hline TG & $140.6 \pm 89$ & $35-800$ \\
\hline HDL & $48.9 \pm 13$ & $18-134$ \\
\hline LDL & $91.6 \pm 31$ & $26-244$ \\
\hline FBS & $171 \pm 73$ & $45-395$ \\
\hline Creatinine & $0.72 \pm 0.22$ & $0-1.6$ \\
\hline
\end{tabular}

$15.9 \mathrm{~mm} \mathrm{Hg}$ at baseline vs. $134 \pm 15.7 \mathrm{~mm} \mathrm{Hg}$ at 6 months, $\mathrm{P}=$ 0.002). However, there was no significant change in DBP after 6 months of treatment $(73.7 \pm 11 \mathrm{~mm} \mathrm{Hg}$ pretreatment vs. $72.7 \pm 10$ $\mathrm{mm} \mathrm{Hg}, \mathrm{P}=0.10)$. The changes in triglycerides and high-density lipoprotein (HDL) were not statistically significant. However, there was a significant reduction in total cholesterol and LDL levels. The total cholesterol dropped from $165 \pm 41 \mathrm{mg} / \mathrm{dL}$ at baseline to $159 \pm 38 \mathrm{mg} / \mathrm{dL}$ after 6 months of treatment $(\mathrm{P}=0.008)$. LDL cholesterol dropped significantly from $91.8 \pm 37 \mathrm{mg} / \mathrm{dL}$ at baseline 


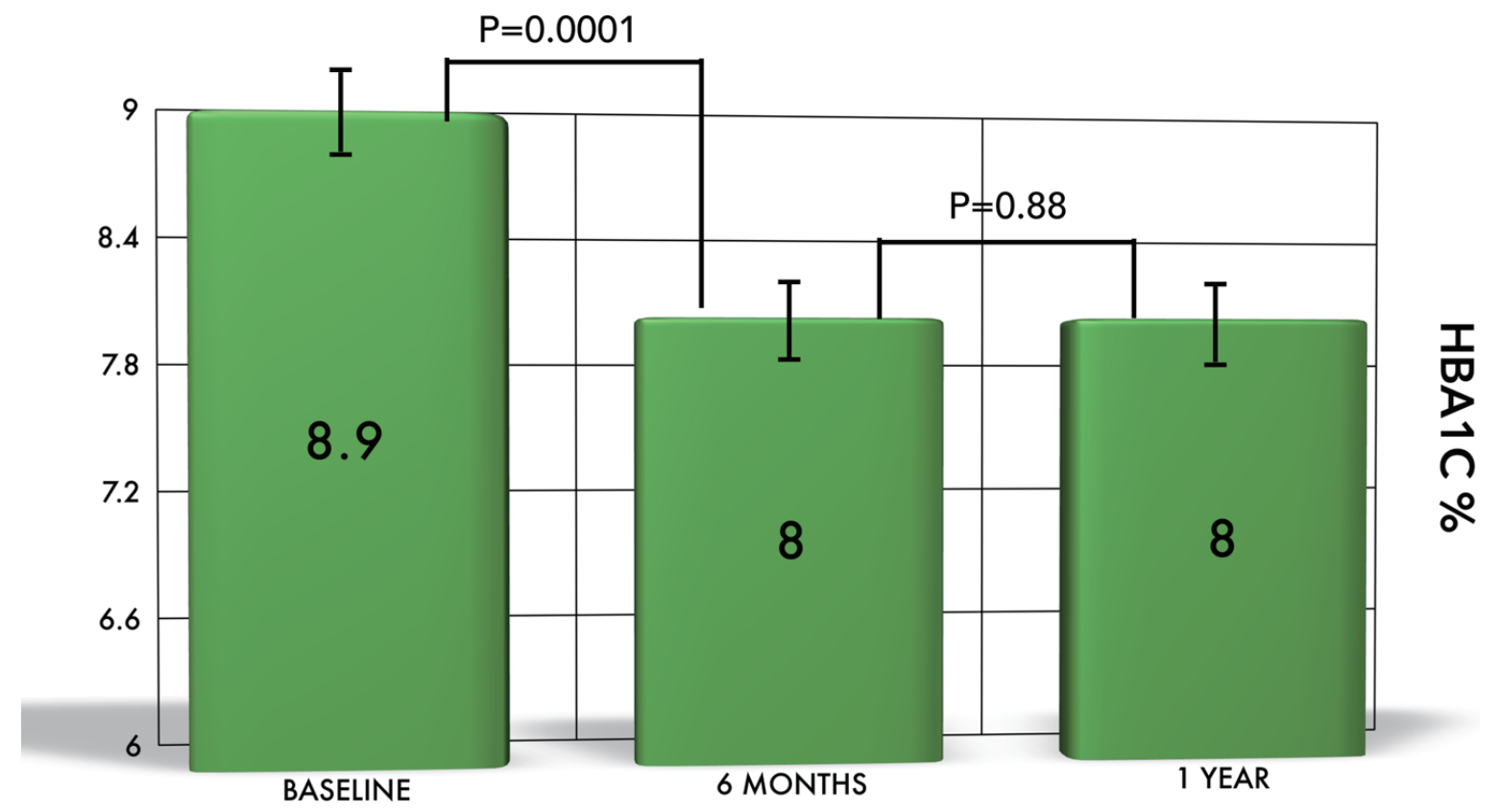

Figure 2. The change of $\mathrm{HbA} 1 \mathrm{c}$ in Emirati patients. There is a significant reduction in $\mathrm{HbA} 1 \mathrm{c}$ from baseline to 6 months and 1 year. However, the difference was not significant between 6 months and 1 year.

to $85.5 \pm 31 \mathrm{mg} / \mathrm{dL}$ at 6 months after treatment $(\mathrm{P}=0.005)$.

\section{Safety and adverse events}

We assessed the adverse events and looked specifically at urinary tract infections, genital infections, and severe hypoglycemia requiring admission. There were only eight reported drugrelated side effects, but none of them required discontinuation of the treatment. All of the patients were females, there were three cases of genital infection treated with a single dose of anti-mycotic agents, and the remaining five cases were urinary tract infections treated with a course of antibiotics. No recurrent urinary tract infections were reported. The overall percentage of reported side effects was $1.9 \%$. We did not observe any case of severe hypoglycemia.

\section{Discussion}

Type 2 diabetes is a chronic progressive disease with multiple associated complications, and it has been a focus of active clinical and therapeutic research for many decades. Fortunately, there have been numerous medical advances made in this regard, such as the discovery of SGLT2 inhibitors. This class of medicine has brought new hope for patients with diabetes and clinicians treating this condition due to the remarkable glycemic and non-glycemic benefits.

Our study shows encouraging results with significant reductions of HBA1c from a mean of $8.9 \pm 1.7 \%$ to $8 \pm 1.5 \%$ at 6 months. This effect was independent of the duration of diabetes, age, gender, other comorbid conditions, and most importantly, other medicines in use. Similarly, FBG improved from
$174 \pm 61 \mathrm{mg} / \mathrm{dL}$ at baseline to $150 \pm 50 \mathrm{mg} / \mathrm{dL}$ at 6 months $(\mathrm{P}<0.0001)$. This highly significant drop in HbA1c has been seen before in many studies and depicts the unique mechanism of action by SGLT2 inhibitors, which do not need functioning beta islets cells [11].

This effect has been seen before in multiple studies in different groups of patients varying from drug-naive subjects with type 2 diabetes to patients on various medications, including insulin. Ferrannini and colleagues reported the efficacy of dapagliflozin as a monotherapy compared to placebo in patients who were inadequately controlled for lifestyle modification. They showed a drop in HbA1c that was highly significant compared to the placebo group for $5 \mathrm{mg}$ of dapagliflozin $(\mathrm{P}<$ $0.001)$ and a $10-\mathrm{mg}$ dose $(\mathrm{P}<0.0001)$ [12]. This effect is seen in other studies involving drug-naive patients. List et al examined the effect of dapagliflozin and metformin in a doubleblind, parallel group and placebo-controlled randomized trial of 389 drug-naive patients. They demonstrated a greater reduction of HBA1c (0.5-0.9\%), FPG (6 - $30 \mathrm{mg} / \mathrm{dL})$, and weight $(2.5-3.4 \%)$ in the dapagliflozin group than in the metformin and placebo groups [13].

Dapagliflozin has been studied for its efficacy as an adjunctive therapy with other oral hypoglycemic agents in different groups of patients. Bailey et al performed a doubleblind placebo-controlled trial of 546 patients on metformin $1,500 \mathrm{mg}$ per day with a mean HBA1c of $7.92 \%$ (although the study was inadequately controlled). Patients were assigned to a placebo group with metformin or a group treated with dapagliflozin at $2.5,5$, or $10 \mathrm{mg}$ per day and metformin and observed for 24 weeks. A statistically significant reduction in HBA1c was observed in the dapagliflozin groups: $-0.67 \%$ $(-0.81$ to $-0.53, \mathrm{P}=0.0002)$ in the dapagliflozin $2.5 \mathrm{mg}$ group, $-0.70 \%(-0.85$ to $-0.56, \mathrm{P}<0.0001)$ in the dapagliflozin $5 \mathrm{mg}$ 


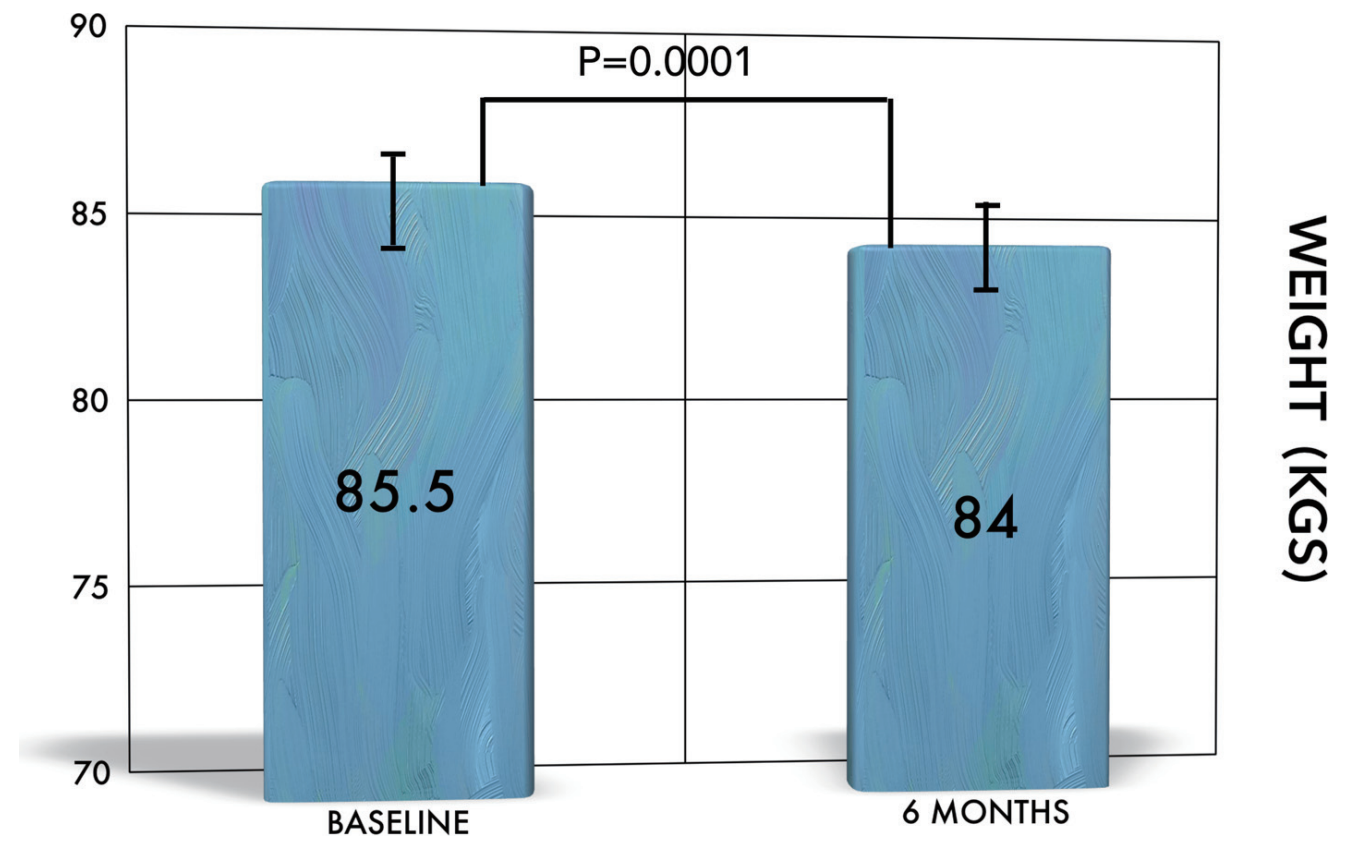

Figure 3. The change of weight over time. There is significant reduction in weight from baseline to 6 months.

group, and $-0.84 \%(-0.98$ to $-0.70, \mathrm{P}<0.0001)$ in the dapagliflozin $10 \mathrm{mg}$ group $(\mathrm{P}<0.001)$. In the metformin and placebo group, the $\mathrm{HbAlc}$ reduction was $-0.30 \%$ (95\% CI: -0.44 to $-0.16)$. A reduction in FPG was also significant in the dapagliflozin group $(17.8-23.4 \mathrm{mg} / \mathrm{dL}, \mathrm{P}<0.002)$ compared to metformin group $(5.9 \mathrm{mg} / \mathrm{dL})$. Weight loss was highly significant in the dapagliflozin group $(2.2-3.0 \mathrm{~kg})$ compared to the metformin group $(0.9 \mathrm{~kg}, \mathrm{P}<0.0001)$. Extension of this trial for 102 weeks in $71.2 \%$ of the original patients in the dapagliflozin group showed a further continuous reduction in all these parameters [14].

SGLT2 inhibitors' unique clinical significance lies in the fact that they can be an adjunctive therapy with any other concurrent hypoglycemic therapies, including insulin. Furthermore, they can be used at any stage of the disease, regardless of the duration of diabetes. There is adequate data available in this regard that strengthen the findings of our study.

SGLT2 inhibitor therapy along with sulfonylurea was initially a concern for the risk of hypoglycemia, and studies have been done to examine the risk associated with this combination. Strojek and colleagues compared the addition of dapagliflozin to $4 \mathrm{mg}$ of glimepiride versus a placebo with glimepiride. They showed a significant reduction in HbA1c after 24 weeks in the dapagliflozin group $(0.58-0.82 \%)$ versus $-0.13 \%$ in the glimepiride and placebo group $(0.13 \%, \mathrm{P}<0.0001)$. Similarly, FPG in the dapagliflozin group showed a considerable reduction $(16.8-28.5 \mathrm{mg} / \mathrm{dL})$ versus the other group $(2.0 \mathrm{mg} / \mathrm{dL})$ over 24 weeks. In regard to hypoglycemia, events were seen in $4.8 \%$ of the placebo group versus $7.1 \%$ of the dapagliflozin group [15].

The change in weight $(1.5 \mathrm{~kg})$ in our cohort was highly significant when the baseline value $(85.7 \pm 17 \mathrm{~kg})$ was compared to that at 6 months $(84.2 \pm 16.6 \mathrm{~kg}, \mathrm{P}=0.0001)$. A similar highly significant change was observed when comparing baseline weight in the Emirati population $(85.7 \pm 17.8 \mathrm{~kg}$ vs. $84 \pm 17.2 \mathrm{~kg}, \mathrm{P}=0.0001)$. This effect is also a consistent finding in previous data.

Clar and colleagues systematically reviewed seven trials with dapagliflozin and one trial with canagliflozin. The metaanalysis showed an average reduction of $\mathrm{HbA} 1 \mathrm{c}$ by $0.54 \%$ by dapagliflozin compared to placebo. The trial with canagliflozin showed slightly more reduction in HbA1c compared to sitagliptin $(0.21 \%)$, but both of these agents resulted in consistent weight loss. Dapagliflozin reduced weight by $1.81 \mathrm{~kg}$ and canagliflozin reduced it by up to $2.3 \mathrm{~kg}$ [16].

Another recent systemic review and meta-analysis examined 13 trials: six trials of dapagliflozin, three trials of canagliflozin, and one for luseogliflozin, ipragliflozin, and tofogliflozin [17]. The results showed that all SGLT2 inhibitors in monotherapy, dual, or triple therapy are more efficient in achieving HbA1c $<7 \%$. Similar to our study, a weight reduction of up to $2.5 \mathrm{~kg}$ was observed. Pacou and colleagues carried out a systemic Bayesian network analysis of 18 trials in 2015. They examined and compared the combination of canagliflozin and metformin as an add-on to insulin with or without another anti-hyperglycemic in uncontrolled patients with type 2 diabetes. This combination was compared with DDP-4 inhibitors, pioglitazone, GLP-1 analogues, dapagliflozin, and sulfonylureas. The outcomes of HbAlc, weight, and hypoglycemia at 26, 52, and 104 weeks were recorded. The results showed that the combination of canagliflozin and metformin with insulin led to a higher $\mathrm{HbA} 1 \mathrm{c}$ reduction compared to sulfonylurea, DPP-4 inhibitors, and dapagliflozin with less hypoglycemia compared to sulfonylureas [18]. This study observed a comparable weight reduction of canagliflozin to GLP analogues. Furthermore, it reinforces the results of our study, where SGLT2 inhibitors were used as dual or triple therapy with or without insulin, with meaningful and consistent 
HbA1c and weight reduction irrespective of age, gender, and concurrent medications. The CANTATA-SU trial was a randomized, double-blind trial of 52-week duration that compared glimepiride with canagliflozin. The study showed a superior reduction of $\mathrm{HbAlc}$ when using $300 \mathrm{mg}$ of canagliflozin compared to glimepiride [19].

Cardiovascular protective effects of SGLT2 inhibitors have been documented since the release of the EMPA REG trial. This effect is not entirely understood. However, the reduction in SBP and DBP, change in oxidative markers, osmotic diuresis, and change in sympathetic tone may play a role. The beneficial effect of SGLT inhibitors on BP has been observed before the release of any cardiovascular outcome trial.

Our results showed a significant reduction in SBP when comparing pre- and post-treatment BPs. The SBP was reduced from $169 \pm 59 \mathrm{~mm} \mathrm{Hg}$ to $147 \pm 50 \mathrm{~mm} \mathrm{Hg}(\mathrm{P}=0.0001)$. A less significant change was observed in DBP, with the baseline DBP of $74.4 \pm 12 \mathrm{~mm} \mathrm{Hg}$ dropping to $73.2 \pm 10 \mathrm{~mm} \mathrm{Hg}$ (P = 0.04). A meta-analysis that included 21 placebo-controlled trials found a mean decrease of $3.77 \mathrm{~mm} \mathrm{Hg}$ in SBP and $1.75 \mathrm{~mm}$ $\mathrm{Hg}$ in DBP in 16 of the trials [20].

Our results showed a statistically significant reduction in total cholesterol and LDL. The unique finding of a $-6.3 \mathrm{mg} / \mathrm{dL}$ reduction in LDL level from $91.8 \pm 37 \mathrm{mg} / \mathrm{dL}$ at baseline to 85.5 $\pm 31 \mathrm{mg} / \mathrm{dL}(\mathrm{P}=0.005)$ has not been observed before. Stenlof et al compared the efficacy of canagliflozin at $100 \mathrm{mg}$ or 300 $\mathrm{mg}$ versus placebo in a double-blind, placebo-controlled randomized trial over 26 weeks. They studied changes in HbAlc, FPG, body weight, SBP, and fasting lipids. Along with a significant reduction in $\mathrm{HbA1c}, \mathrm{FPG}$, and weight, their results also showed a decrease in SBP (-1.4 and $3.9 \mathrm{~mm} \mathrm{Hg})$, but there was no change in DBP as in our study. Lipid parameters showed increased HDL and LDL and decreased triglycerides [21].

This effect does not seem to be related to ethnicity, as a similar effect by canagliflozin was seen in a randomized placebo-controlled double blind trial in 676 Asian patients by Ji et al. They observed the effect of canagliflozin in patients with type 2 diabetes (although inadequately controlled), who were on metformin or metformin with a sulfonylurea. They found a slight increase in LDL and HDL and a decrease in triglycerides in the canagliflozin group. Furthermore, there was a change in SBP of $3.1 \mathrm{~mm} \mathrm{Hg}$ for $100 \mathrm{mg}$ of canagliflozin and up to 3.4 $\mathrm{mm} \mathrm{Hg}$ in the 300-mg dose group over 18 weeks [22].

In a recent review, Inzucchi and colleagues reviewed 12 trials of dapagliflozin involving 3,731 patients with a mean duration of 24 weeks. The results showed a mean increase in LDL by $0.6 \%$ and $2.7 \%$ from baseline with $5-\mathrm{mg}$ and $10-\mathrm{mg}$ doses of dapagliflozin, respectively. Similarly, four trials of empagliflozin involving 2,700 patients using $10-\mathrm{mg}$ and $25-$ $\mathrm{mg}$ doses showed increases in LDL by $0.08 \%$ and $0.10 \%$, respectively [20]. We did not find any study on any ethnic group that found a reduction in LDL level, as observed in our study. This effect needs further evaluation in larger randomized controlled trials.

\section{Safety data}

We observed the incidence of acute kidney injury, severe hy- poglycemia, and genitourinary infection in the cohort of patients after starting SGLT2 inhibitors. Our results showed no cases of acute kidney injury; three cases of genital mycosis, which resolved after topical anti-mycotic therapy; and five cases of urinary tract infection, which responded well to oral antibiotics. All these adverse effects were seen in female patients, and none of them discontinued SGLT2 inhibitors due to side effects. The incidence of genital infection seems to be lower in our group compared to international data. Data from a recent review that included 12 dapagliflozin trials compared the safety of dapagliflozin (4,545 patients) versus a placebo (1,393 patients). The study showed higher incidences of UTI $(7.3 \%$ and $6.5 \%)$ in the groups receiving $10 \mathrm{mg}$ and $5 \mathrm{mg}$ of dapagliflozin, respectively, versus $4.5 \%$ in the placebo group. Genital mycotic infections also had higher incidence in patients treated with dapagliflozin $(4.1-5.7 \%$ versus $0.9 \%)$ in the placebo group [17].

The CANATA-SU trial showed genital mycotic infection in $11 \%$ (26 patients) with the $100-\mathrm{mg}$ dose and 14\% (34 patients) of females in the $300-\mathrm{mg}$ dose group. Genital mycotic infections were seen in 14\% (34 patients) versus $2 \%$ (five patients) in the glimepiride group. Among males using $300 \mathrm{mg}$ and $100 \mathrm{mg}$ of canagliflozin, 7\% (17 patients) and 8\% (20 patients) showed infections, respectively. UTI occurred in 6\% (31 patients) for both canagliflozin doses versus 5\% (22 patients) in the glimepiride group. The canagliflozin group had fewer side effects (5\%) compared to the glimepiride group (8\%) [19].

In their systematic review and meta-analysis, Vasilakou et al showed that SGLT2 inhibitors had a higher incidence of genitourinary infection and a similar incidence of hypoglycemia to other active comparators [2]. However, in our cohort, the risk of genitourinary infections was much lower than international figures. Moreover, we did not observe any case of severe hypoglycemia, regardless of the baseline medication used.

\section{Limitations}

This is a retro-prospective study and has the typical limitations of this type of investigation. However, the total number of patients included is significant and gives the study the power to assess these variables.

\section{Conclusion}

The use of SGLT2 inhibitors is associated with significant reductions in $\mathrm{HbA1c}$ and weight. However, unlike in all previous trials, the inhibitors reduced total cholesterol and LDL significantly. Larger trials are needed to reassess the effects on lipid parameters.

\section{Acknowledgments}

The authors would like to thank the DHA ethical committee for their ongoing support to researchers in Dubai. We would 
also thank Mr. Fahad and Mrs. Badriya from the medical records section of Dubai Hospital for their ongoing support and help in providing medical charts in a timely and organized manner.

\section{Author Contributions}

Alaaeldin Bashier carried out the design, participated in data collection, data revalidation, data analysis, and manuscript writing, and carried out major review and final review of the manuscript. Azza Abdulaziz Khalifa participated in data collection, data revalidation, and review of the manuscript. Fauzia Rashid participated in manuscript writing, review of the manuscript. Elamin Ibrahim Abdelgadir participated in manuscript review. Amina Adil Al Qaysi participated in data collection. Razan Ali participated in data collection. Ahmed Eltinay participated in data collection. Jalal Nafac participated in manuscript review. Fatima Alsayyah participated in data collection. Fatheya Alawadi participated in manuscript review. All authors approved the manuscript.

\section{Funding}

No funds were received.

\section{Competing Interests}

The authors declare no competing interests.

\section{Consent}

Available for those who were followed prospectively.

\section{Ethical Committee Approval}

The DHA ethical committee approved the study.

\section{References}

1. Mikhail N. Place of sodium-glucose co-transporter type 2 inhibitors for treatment of type 2 diabetes. World J Diabetes. 2014;5(6):854-859.

2. Vasilakou D, Karagiannis T, Athanasiadou E, Mainou M, Liakos A, Bekiari E, Sarigianni M, et al. Sodiumglucose cotransporter 2 inhibitors for type 2 diabetes: a systematic review and meta-analysis. Ann Intern Med. 2013;159(4):262-274.

3. Wilding JP, Woo V, Rohwedder K, Sugg J, Parikh S. Dapagliflozin in patients with type 2 diabetes receiving high doses of insulin: efficacy and safety over 2 years. Diabetes Obes Metab. 2014;16(2):124-136.

4. Baker WL, Smyth LR, Riche DM, Bourret EM, Chamber- lin KW, White WB. Effects of sodium-glucose co-transporter 2 inhibitors on blood pressure: a systematic review and meta-analysis. J Am Soc Hypertens. 2014;8(4):262275 e269.

5. Zinman B, Wanner C, Lachin JM, Fitchett D, Bluhmki E, Hantel S, Mattheus M, et al. Empagliflozin, Cardiovascular Outcomes, and Mortality in Type 2 Diabetes. N Engl J Med. 2015;373(22):2117-2128.

6. Wanner C, Inzucchi SE, Lachin JM, Fitchett D, von Eynatten M, Mattheus M, Johansen OE, et al. Empagliflozin and Progression of Kidney Disease in Type 2 Diabetes. N Engl J Med. 2016;375(4):323-334.

7. Yang W, Ji L, Zhou Z, Cain VA, Johnsson KM, Sjostrom CD. Efficacy and safety of dapagliflozin in Asian patients: A pooled analysis. J Diabetes. 2016.

8. Kaku K, Kiyosue A, Inoue S, Ueda N, Tokudome T, Yang J, Langkilde AM. Efficacy and safety of dapagliflozin monotherapy in Japanese patients with type 2 diabetes inadequately controlled by diet and exercise. Diabetes Obes Metab. 2014;16(11):1102-1110.

9. Ji L, Ma J, Li H, Mansfield TA, T'Joen C L, Iqbal N, Ptaszynska A, et al. Dapagliflozin as monotherapy in drug-naive Asian patients with type 2 diabetes mellitus: a randomized, blinded, prospective phase III study. Clin Ther. 2014;36(1):84-100 e109.

10. Gavin JR, 3rd, Davies MJ, Davies M, Vijapurkar U, Alba $\mathrm{M}$, Meininger $\mathrm{G}$. The efficacy and safety of canagliflozin across racial groups in patients with type 2 diabetes mellitus. Curr Med Res Opin. 2015;31(9):1693-1702.

11. Anderson SL. Dapagliflozin efficacy and safety: a perspective review. Ther Adv Drug Saf. 2014;5(6):242-254.

12. Ferrannini E, Ramos SJ, Salsali A, Tang W, List JF. Dapagliflozin monotherapy in type 2 diabetic patients with inadequate glycemic control by diet and exercise: a randomized, double-blind, placebo-controlled, phase 3 trial. Diabetes Care. 2010;33(10):2217-2224.

13. List JF, Woo V, Morales E, Tang W, Fiedorek FT. Sodium-glucose cotransport inhibition with dapagliflozin in type 2 diabetes. Diabetes Care. 2009;32(4):650-657.

14. Bailey CJ, Gross JL, Pieters A, Bastien A, List JF. Effect of dapagliflozin in patients with type 2 diabetes who have inadequate glycaemic control with metformin: a randomised, double-blind, placebo-controlled trial. Lancet. 2010;375(9733):2223-2233.

15. Strojek K, Yoon KH, Hruba V, Elze M, Langkilde AM, Parikh S. Effect of dapagliflozin in patients with type 2 diabetes who have inadequate glycaemic control with glimepiride: a randomized, 24-week, doubleblind, placebo-controlled trial. Diabetes Obes Metab. 2011;13(10):928-938.

16. Clar C, Gill JA, Court R, Waugh N. Systematic review of SGLT2 receptor inhibitors in dual or triple therapy in type 2 diabetes. BMJ Open. 2012;2(5).

17. Shyangdan DS, Uthman OA, Waugh N. SGLT-2 receptor inhibitors for treating patients with type 2 diabetes mellitus: a systematic review and network meta-analysis. BMJ Open. 2016;6(2):e009417.

18. Taieb V, Pacou M, Schroeder M, Nielsen AT, Neslusan C, Schubert A. Bayesian Network Meta-Analysis (NMA) to 
Assess the Relative Efficacy of Canagliflozin in Patients with Type 2 Diabetes Mellitus (T2DM) Inadequately Controlled with Insulin. Value Health. 2015;18(7):A598.

19. Cefalu WT, Leiter LA, Yoon KH, Arias P, Niskanen L, Xie J, Balis DA, et al. Efficacy and safety of canagliflozin versus glimepiride in patients with type 2 diabetes inadequately controlled with metformin (CANTATA-SU): 52 week results from a randomised, double-blind, phase 3 non-inferiority trial. Lancet. 2013;382(9896):941-950.

20. Inzucchi SE, Zinman B, Wanner C, Ferrari R, Fitchett D, Hantel S, Espadero RM, et al. SGLT-2 inhibitors and cardiovascular risk: proposed pathways and review of ongo- ing outcome trials. Diab Vasc Dis Res. 2015;12(2):90-100.

21. Stenlof K, Cefalu WT, Kim KA, Jodar E, Alba M, Edwards $\mathrm{R}$, Tong $\mathrm{C}$, et al. Long-term efficacy and safety of canagliflozin monotherapy in patients with type 2 diabetes inadequately controlled with diet and exercise: findings from the 52-week CANTATA-M study. Curr Med Res Opin. 2014;30(2):163-175.

22. Ji L, Han P, Liu Y, Yang G, Dieu Van NK, Vijapurkar U, Qiu R, et al. Canagliflozin in Asian patients with type 2 diabetes on metformin alone or metformin in combination with sulphonylurea. Diabetes Obes Metab. 2015;17(1):23-31. 